\title{
COMPOSICIÓN QUÍMICA Y ACTIVIDAD ANTIMICROBIANA DEL ACEITE ESENCIAL de Pelargonium odoratissimum (L) L'Hér (Geraniaceae)
}

Fecha de recepción: 28 de julio de 2015• Fecha de Evaluación: 07 de marzo 2016 • Fecha de aceptación: 17 de marzo de 2016

\section{CHEMICAL COMPOSITION AND ANTIMICROBIAL ACTIVITY OF ESSENTIAL OIL OF Pelargonium odoratissimum (L) L'Hér (Geraniaceae)}

Luis Miguel Pombo Ospina ${ }^{1,3}$, Javier A. Matulevich Peláez², Paola Borrego Muñoz¹, William F. Castrillón Cardona², Leidy Barajas Villamizar ${ }^{1}$.

\section{RESUMEN}

La especie Pelargonium odoratissimum (geranio de olor) pertenece a la familia Geraniaceae. Dentro de su composición química, presenta flavonoides tales como quercetina, kaempferol y miricetina; el aceite esencial de sus hojas es rico en metil-eugenol, limoneno y fencona. A partir de hojas y flores de la especie $P$. odoratissimum se obtuvo el aceite esencial por hidrodestilación (rendimiento 0,3\% m/v); la composición química se determinó por cromatografía de gases acoplada a espectrometría de masas (CG-EM), comparando los índices de retención y los espectros de masas con los datos reportados en la literatura. Se determinó la presencia de 4 monoterpenos, 20 sesquiterpenos y algunos compuestos oxigenados entre ellos 7 ésteres y un ácido, los cuales constituyen cerca del $74 \%$ de la composición relativa total del aceite. Los monoterpenos identificados representan el 22,60\% de la composición del aceite esencial, en los cuales los componentes mayoritarios encontrados fueron geraniol (12,69\%) y citronelol (8,99\%). La actividad antimicrobiana medida como la Concentración Mínima Inhibitoria (CMI) se realizó por el método de microdilución en placas de 96 pozos utilizando como indicador de viabilidad el MTT (bromuro de 3-[4,5 dimetiltiazol-2-il]-2,5-difeniltetrazolio). El aceite esencial mostró actividad frente a todas las cepas microbianas ensayadas. La mayor actividad se encuentra frente a Staphylococcus aureus, Proteus mirabilis, Aspergillus brasiliensis y Candida albicans con una $\mathrm{CMI}<3,9 \mu \mathrm{g} / \mathrm{mL}$. Se encontró una menor actividad inhibitoria, aunque no menos importante, contra Trichophytum rubrum $(\mathrm{CMI}=62,5 \mu \mathrm{g} / \mathrm{mL})$ y Trichophytum mentagrophytes $(\mathrm{CMI}=125 \mu \mathrm{g} / \mathrm{mL})$.

Palabras Clave: Pelargonium odoratissimum, Aceite esencial, CG-EM, Actividad antimicrobiana.

1 Grupo de Investigación en Farmacología Vegetal y Terapéuticas Alternativas (GIFVTA), Departamento de Ciencias Básicas. Facultad de Medicina. Fundación Universitaria Juan N. Corpas. Bogotá, Colombia.

2 Grupo de Productos Naturales Vegetales. Proyecto Curricular de Licenciatura en Química, Facultad de Ciencias y Educación. Universidad Distrital Francisco José de Caldas. Bogotá, Colombia.

3 Autor Corresponsal. E-mail: miguel.pombo@juanncorpas.edu.co 


\section{ABSTRACT}

Pelargonium odoratissimum species (Apple pelargonium) belongs to the family Geraniaceae. In their chemical composition, flavonoids such as quercetin, kaempferol and myricetin are present, the essential oil of the leaves is rich in methyl eugenol, limonene and fenchone. From leaves and flowers of the species $P$. odoratissimum, the essential oil was obtained by hydrodistillation (yield of $0.3 \% \mathrm{~m} / \mathrm{v}$ ); the chemical composition was determined by gas chromatography coupled to mass spectrometry (GC-MS), comparing the retention index and mass spectral with the data reported in the literature. It was determined the presence of 4 monoterpenes, 20 sesquiterpenes and some oxygenated compounds including 7 esters and one acid, which constitute about $74 \%$ of the total oil relative composition. The identified monoterpenes represent $22,60 \%$ of the composition of the essential oil, in which the main components founded were geraniol (12,69\%) and citronellol (8,99\%). The antimicrobial activity measured as the Minimum Inhibitory Concentration (MIC) was made by the microdilution method in microplates of 96 wells using the MTT as indicator of the viability. (3-[4,5 dimethylthyazol-2-yl] -2,5-diphenyltetrazolium bromide). The essential oil showed activity in presence of all tested microbial strains. The highest activity was found for Staphylococcus aureus, Proteus mirabilis, Aspegillus brasiliensis and Candida albicans with a MIC $<3.9 \mu \mathrm{g} / \mathrm{mL}$. It was found less inhibitory activity, but not least, against Trichophytum rubrum ( $\mathrm{MIC}=62.5 \mu \mathrm{g} / \mathrm{mL}$ ) and Trichophytum mentagrophytes $(\mathrm{MIC}=125 \mu \mathrm{g} / \mathrm{mL})$.

Key Words: Pelargonium odoratissimum, Essential oil, GC-MS, Antimicrobial activity.

\section{INTRODUCCIÓN}

De acuerdo con la Organización Mundial de la Salud (OMS), gran diversidad de especies vegetales han sido empleadas como fuente principal para el desarrollo de fitomedicamentos. Estos desarrollos han tomado como base algunos estudios etnobotánicos que sustentan el uso tradicional de las plantas medicinales de una región en particular. El uso incorrecto de fármacos antibacterianos ha favorecido la selección y propagación de bacterias resistentes y en consecuencia, los fármacos antibacterianos han perdido gradualmente su efectividad, lo que ha desencadenado una emergencia sanitaria a nivel mundial (WHO, 2014).

Los aceites esenciales (AE), extractos y fracciones obtenidos a partir de algunas especies vegetales presentan actividades antibacterial, antifúngica y antiviral y han sido estudiados como nueva fuente de compuestos antimicrobianos como alternativa para el tratamiento de enfermedades infecciosas (Solórzano y Miranda, 2012; Alviano, 2009). La hidrofobicidad de los AE y sus componentes, les permite interactuar de manera eficiente con los lípidos de las membranas celular y mitocondrial de las bacterias, alterando la permeabilidad de éstas estructuras (Lorenzi et al, 2009; Devi et al, 2010). Dentro de los compuestos más estudiados por su actividad antimicrobiana presentes en los $A E$, se encuentran el cinamaldehído, el geraniol, el timol, el mentol y el carvacol (Solórzano y Miranda, 2012; Langeveld et al, 2014).

P. odoratissimun (geranio de olor) pertenece a la familia Geraniaceae, sus hojas son sencillas, simétricas, con el ángulo basal ampliamente obtuso y pecíolo basal. El género Pelargonium, cuenta con cerca de 250 especies, de las cuales el $80 \%$ se 
producen en el sur de África y el resto en el este de África, Madagascar y Australia (Romitelli y Martins, 2013). En su composición química, presenta flavonoides tales como quercetina, kaempferol y miricetina y el aceite esencial de sus hojas es rico en metil-eugenol, limoneno y fencona (Andrade et al, 2011). Toda la planta es una hierba aromática con propiedades astringentes, tónicas y efectos antisépticos. Se utiliza internamente en el tratamiento de la debilidad, la gastroenteritis y en el control de hemorragias. Externamente, se usa para tratar afecciones de la piel, lesiones, neuralgias e infecciones de garganta (Bown, 1995). Algunos estudios han reportado que el aceite esencial obtenido a partir de las hojas frescas de $P$. odoratissimun muestra una marcada actividad antimicrobiana frente a diferentes cepas microbianas patógenas (Andrade et al, 2011; Bussmann et al, 2010).

El objetivo principal de este estudio, teniendo en cuenta el bajo volumen de información que se encuentra de la especie estudiada, fue evaluar la actividad antimicrobiana del aceite esencial obtenido a partir de las hojas y las flores de P. odoratissimun y contribuir al conocimiento de su composición química.

\section{MATERIALES Y MÉTODOS}

Hojas y flores frescas de P. odoratissimum, fueron recolectadas en el Jardín Medicinal de la Fundación Universitaria Juan N. Corpas (Altitud. 2554m, $\left.4^{\circ}, 45,722^{\prime} \mathrm{N}, 74^{\circ} 5,644^{\prime} \mathrm{O}\right)$ ubicado en la Sabana de Bogotá (Localidad de Suba). Las muestras fueron recolectadas en mayo de 2015. Todo el material vegetal fue identificado y autenticado por El Herbario Federico Mederm Bogotá, sede Villa de Leyva, con número de voucher AM32D. El aceite esencial fue obtenido empleando el método de hidrodestilación en un equipo Clevenger modificado durante cuatro horas de extracción. Se utilizaron $300 \mathrm{~g}$ de hojas frescas y flores, obteniéndose $0,9 \mathrm{~mL}$ de aceite. La determinación de la composición química del aceite esencial se realizó por CG-EM en un equipo SHIMADZU QP2010 plus, empleando una columna capilar de sílice fundida, HP-5MS (J \& W Scientific, Folsom, CA, EE.UU.) de $60 \mathrm{~m} \times 0,25 \mathrm{~mm} \times 0,25 \mu \mathrm{m}$, con fase estacionaria $5 \%$ fenilpolimetilsiloxano. La programación de temperatura del horno fue de 40 ${ }^{\circ} \mathrm{C}$ (5 min) con incremento de $4{ }^{\circ} \mathrm{C} / \mathrm{min}$, hasta 160 ${ }^{\circ} \mathrm{C}(0 \mathrm{~min})$; incremento de $2,5^{\circ} \mathrm{C} / \mathrm{min}$, hasta $220^{\circ} \mathrm{C}(0$ min) y por ultimo de $8{ }^{\circ} \mathrm{C} / \mathrm{min}$, hasta $280^{\circ} \mathrm{C}(4 \mathrm{~min})$. Los espectros de masas se obtuvieron por impacto electrónico (IE) con energía de 70 eV. Las temperaturas de la cámara de ionización y de la línea de transferencia fueron de $230^{\circ} \mathrm{C}$ y $275^{\circ} \mathrm{C}$, respectivamente; el modo de inyección fue splitless (10:1) y el volumen de inyección de muestra de $1 \mu \mathrm{L}$. El gas de arrastre utilizado fue helio (grado 5.0), con flujo constante de 1,2 $\mathrm{mL} / \mathrm{min}$. Los índices de retención (IR) se calcularon teniendo en cuenta los tiempos de retención de una serie homóloga de patrones de hidrocarburos desde $C_{7}$ hasta $C_{24}$ analizados bajo las mismas condiciones que el aceite esencial.

Para la evaluación de la actividad antimicrobiana, las cepas utilizadas en los ensayos fueron ATCC (American Type Culture Collection), para Gram positivas Staphylococcus aureus ATCC 6538, Streptococcus faecalis ATCC 29212 y Bacillus subtilis ATCC 6633; y para Gram negativas Escherichia coli ATCC 8739; Salmonella typhimurium ATCC 14028, Proteus mirabilis ATCC 43071, para hongos Aspergillus brasiliensis ATCC 16404, Trichophytum rubrum ATCC 28188, Trichophytum mentagrophytes ATCC 9533 y levaduras Candida albicans ATCC 10231. El cultivo celular de cada uno de los microorganismos se preparó inoculando cada una de las cepas ATCC, por siembra masiva en una caja de Petri con Agar Tripticasa de Soya, a partir de las perlas del sistema de preservación Cryobank. Se incubaron a $35^{\circ} \mathrm{C} \pm 2^{\circ} \mathrm{C}$ por 24 horas, condiciones 
para bacterias y a $25^{\circ} \mathrm{C} \pm 2^{\circ} \mathrm{C}$ por 72 horas para hongos. Transcurrido el tiempo de incubación se realizó la tinción de Gram para cada uno de los microorganismos verificando la pureza del cultivo y a través de medios sólidos selectivos. El inoculo utilizado contiene $3,0 \times 10^{8} \mathrm{UFC} / \mathrm{mL}$, equivalentes al estándar número 1 de la escala McFarland (USP, 2014). La Concentración Mínima Inhibitoria (CMI) se realizó por el método de microdilución de placas de 96 pozos. El medio utilizado para las diluciones fue agua peptonada, en cada pozo de la microplaca se adicionaron $100 \mu \mathrm{L}$ de medio de cultivo líquido, y $50 \mu \mathrm{L}$ del $\mathrm{AE}$ ajustando las concentraciones finales del aceite esencial a 500; 250; 62,$5 ; 31,25 ; 15,6 ; 7,8$ y $3,9 \mu \mathrm{g} / \mathrm{mL}$, posteriormente se adicionaron $100 \mu \mathrm{L}$ de la suspensión microbiana con una concentración de $1 \times 10^{6}$ UFC. Las placas fueron incubadas por $24 \mathrm{~h}$ a $35^{\circ} \mathrm{C} \pm 2^{\circ} \mathrm{C}$ para bacterias y para hongos a $25^{\circ} \mathrm{C} \pm 2^{\circ} \mathrm{C}$. Finalmente, se adicionaron $10 \mu \mathrm{L}$ de MTT (bromuro de 3-[4,5dimetiltiazol-2-il]-2,5-difeniltetrazolio) por cada pozo a una concentración de $2,5 \mu \mathrm{g} / \mathrm{mL}$, se incubaron por 8 horas a las mismas temperaturas del proceso de incubación mencionado, la absorbancia se midió a $550 \mathrm{~nm}$ en un lector de Microplacas Elisa Accu Reader M965. La CMI fue definida como la concentración más baja de aceite esencial que inhibe el crecimiento microbiano. Todos los ensayos se realizaron por triplicado (Vega et al, 2013). Se usaron como controles positivos Gentamicina y Anfotericina B.

\section{RESULTADOS Y DISCUSIÓN}

El aceite esencial de hojas y flores de P. odoratissimun fue obtenido como un líquido ligeramente verde, con un rendimiento del 0,3\% (m/v). La identificación de los componentes presentes se realizó comparando los índices de retención y los espectros de masas con datos reportados en la literatura
(Adams, 2007; Goodner, 2008; Babushok y Zenkevich, 2009). Se determinó la presencia de monoterpenos, sesquiterpenos y compuestos oxigenados, entre ellos ésteres y ácidos, los cuales constituyen cerca del $74 \%$ de la composición relativa total del aceite. En la Tabla 1 se encuentran los compuestos que por comparación presentan más de un $90 \%$ de coincidencia con el espectro de la librería NIST 08 y los porcentajes de abundancia de cada componente dentro del aceite esencial.

En el aceite esencial fueron identificados $32 \mathrm{com}$ puestos (73,97\%); 4 monoterpenos, 20 sesquiterpenos, 7 ésteres y un ácido. Los monoterpenos identificados representan el 22,60\% de la composición del aceite esencial, en los cuales los componentes mayoritarios encontrados fueron geraniol $(12,69 \%)$ y citronelol (8,99\%). Los sesquiterpenos encontrados corresponden al $34,42 \%$, siendo $\gamma$-eudesmol $(11,40 \%)$ y bergamoteno $(10,63 \%)$ los componentes mayoritarios. En cuanto a los ésteres encontrados representan el $14,85 \%$ donde el constituyente en mayor porcentaje corresponde a fenil-etil-butanoato $(5,74 \%)$ seguido del acetato de cinamilo (3,32\%). El ácido encontrado corresponde al ácido caprílico (2,1\%).

El análisis comparativo de los componentes identificados en el aceite esencial de P. odoratissimun con los de especies distribuidas en República Checa (Matusinsky et al, 2015) y Brasil (Andrade et al, 2011) permitió identificar compuestos comunes entre estas especies; tal es el caso de algunos monoterpenos como linalol, citronelol y geraniol y sesquieterpenos como $\gamma$-eudesmol, $\alpha$-copaeno y sibireno. En cuanto a los componentes mayoritarios, existen diferencias marcadas, ya que para el caso del aceite extraído en este estudio se identificó el geraniol (12,69\%) como el compuesto con mayor porcentaje, mientras que en el estudio de $P$. odoratissimum originario de la República Checa se reporta el $\beta$-citronelol (24,86\%) como monoterpeno mayoritario seguido del geraniol (12,50\%), y 


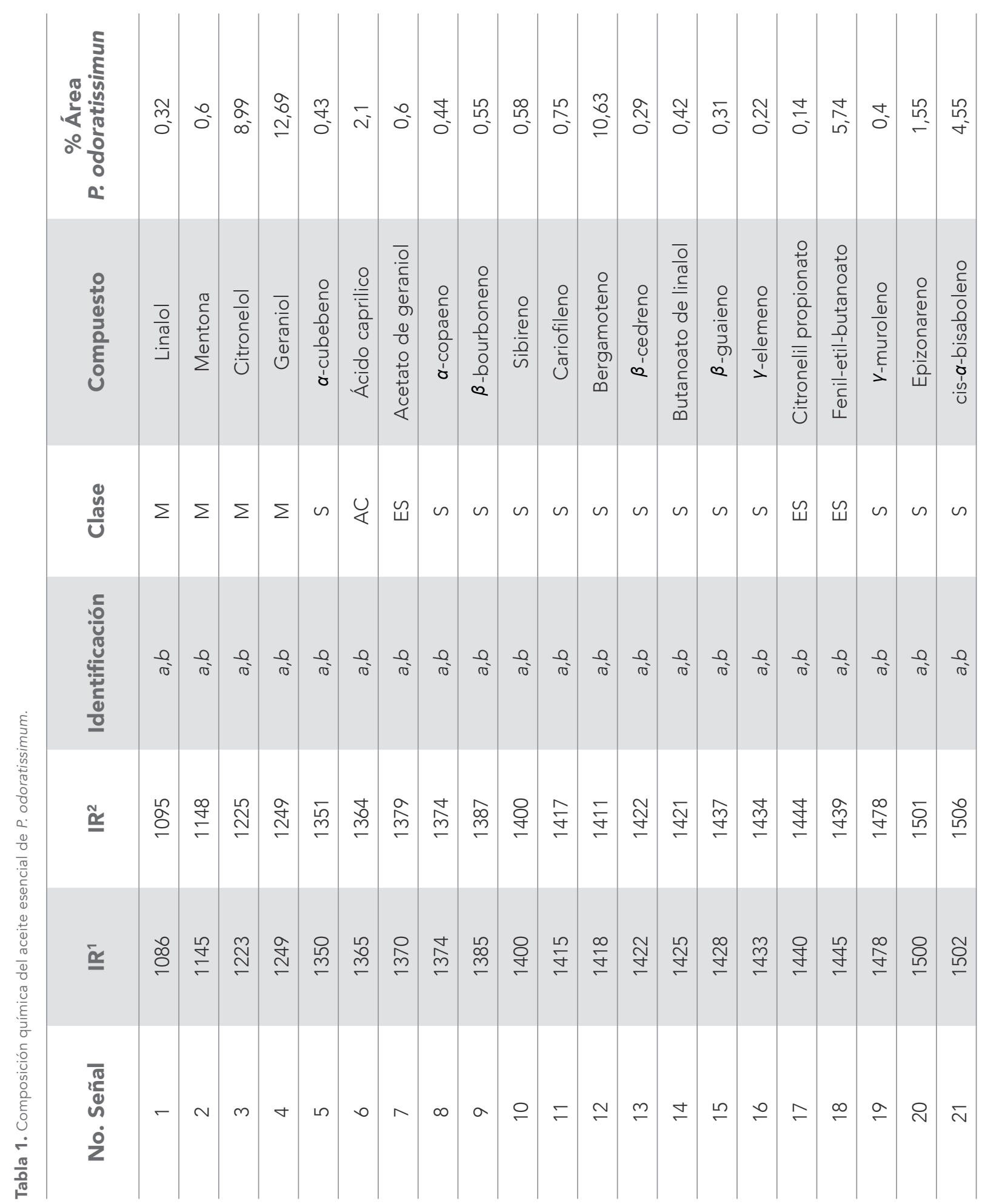




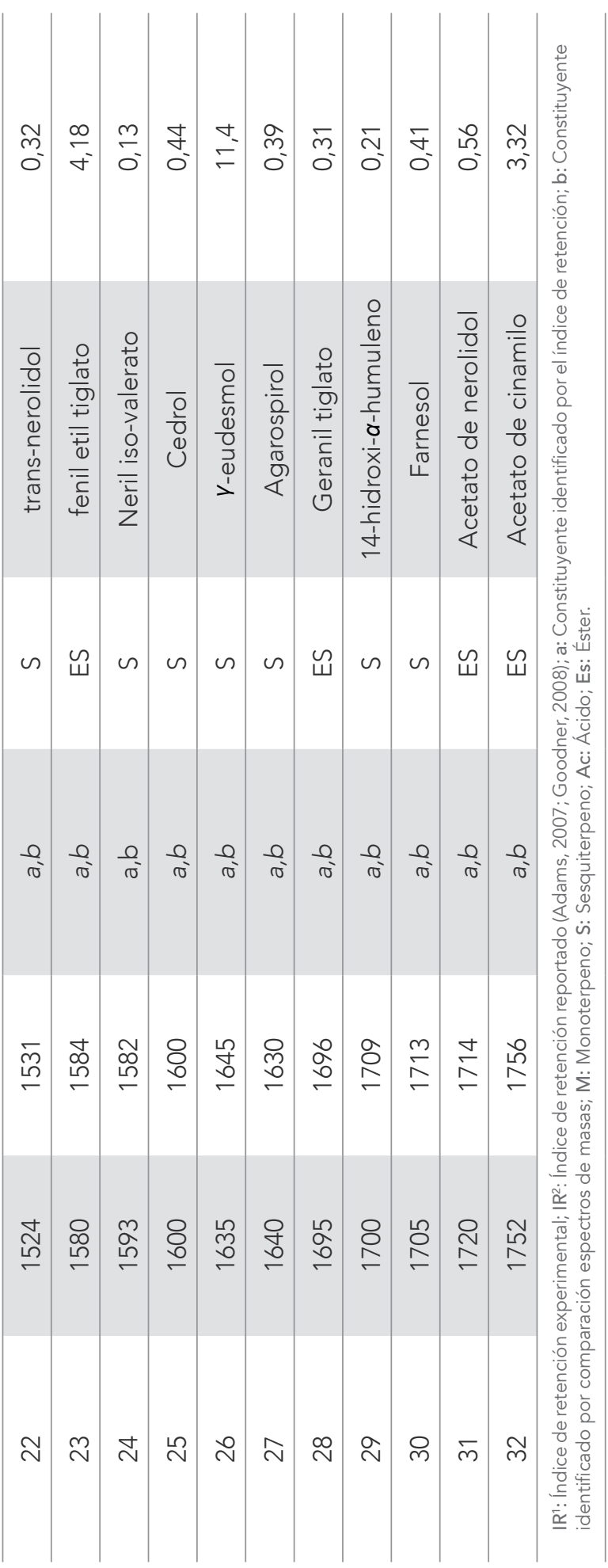

para la especie originaria de Brasil el metileugenol (96,80\%). Posiblemente, las diferencias en composición química de los aceites esenciales pueden estar relacionadas con el hecho de que a pesar de que es la misma especie estudiada, las condiciones ambientales (temperatura, humedad relativa, época de recolección, suelos, etc) son diferentes (Nurzyńska -Wierdak et al, 2012).

La CMI del aceite esencial de las hojas y las flores de $P$. odoratissimun sobre las diferentes cepas microbianas se observa en la Tabla 2. Es importante destacar que el aceite de la especie estudiada mostró actividad frente a todas las cepas microbianas ensayadas. La mayor actividad antimicrobiana sobre microorganismos Gram positivos, se encuentra para $S$. aureus con una $\mathrm{CMl}<3,9 \mu \mathrm{g} / \mathrm{mL}$, éste resultado concuerda con la actividad reportada por Lis et al. (1998). Para microorganismos Gram negativos el mayor efecto inhibitorio se observa frente a $P$. mirabilis con una $\mathrm{CMI}<3,9 \mu \mathrm{g} / \mathrm{mL}$. De igual forma, el aceite fue activo frente a hongos $y$ levaduras, específicamente frente a $A$. brasiliensis y C. albicans con una $\mathrm{CMl}<3,9 \mu \mathrm{g} / \mathrm{mL}$, en ambos casos. Se encontró una menor actividad inhibitoria, aunque no menos importante, para las cepas de hongos resistentes, $T$. rubrum $(\mathrm{CMI}=62,5 \mu \mathrm{g} /$ $\mathrm{mL}$ ) y $\mathrm{T}$. mentagrophytes $(\mathrm{CMI}=125 \mu \mathrm{g} / \mathrm{mL})$. El estudio de Andrade et al., (2011) mostró que el aceite esencial de $P$. odoratissimum fue activo frente a las cepas de los hongos Aspergillus flavus, Aspergillus carbonarius y Aspergillus parasiticus y no evidenció actividad antimicrobiana frente a S. aureus y E. coli; estos resultados difieren de los obtenidos en el presente estudio.

Los resultados de la actividad antimicrobiana observada en este estudio, para el aceite esencial de P. odoratissimum, sobre cepas Gram positivas, Gram negativas, hongos, se pueden explicar por la presencia de los compuestos mayoritarios geraniol y citronelol y adicionalmente, por la presencia de 
algunos otros compuestos que se encuentran en bajas cantidades como el linalol y el cariofileno. Boukhatem et al. (2013), observaron que la actividad de los aceites esenciales obtenidos a partir de especies de Pelargonium, se debe principalmente a la presencia de compuestos mayoritarios como el citronelol y el geraniol; asimismo, Xianfei et al. (2007), reportaron que algunos componentes en bajas cantidades como cimeno, linalol, geranial y cariofileno, contribuyen a la actividad antimicrobiana de los aceites esenciales.

Algunos estudios han demostrado que el geraniol, compuesto con mayor porcentaje de abundancia,
De igual forma, la fuerte actividad del aceite esencial del geranio de olor, frente a C. albicans, puede estar relacionada con la presencia de geraniol, geranil acetato y citronelol, de acuerdo con los resultados obtenidos por Zore et al. (2011), que a su vez asocian ésta actividad con la alteración de la integridad de la membrana y la inhibición del tubo germinal y el ciclo celular de esta levadura.

Un gran volumen de literatura da soporte al hecho de que los AE obtenidos por diferentes técnicas, puros o en combinación con agentes antimicrobianos sintéticos, son una fuente importante de preparaciones farmacéuticas con actividad

Tabla 2. Concentración mínima inhibitoria (CMI) del aceite de P. odoratissimum sobre las diferentes cepas microbianas.

\begin{tabular}{|c|c|c|c|}
\hline Tipo de microorganismo & Cepas & CMI $(\mu \mathrm{g} / \mathrm{mL})$ & $\begin{array}{c}\text { CMI Controles positi- } \\
\text { vos }(\mu \mathrm{g} / \mathrm{mL})\end{array}$ \\
\hline \multirow{3}{*}{ Gram positivos } & Staphylococcus aureus & $<3,9$ & $1,5^{\mathrm{a}}$ \\
\hline & Streptococcus faecalis & 62,5 & $1,6^{\mathrm{a}}$ \\
\hline & Bacillus subtilis & 15,6 & $1,3^{\mathrm{a}}$ \\
\hline \multirow{3}{*}{ Gram negativas } & Escherichia coli & 15,6 & $0,28^{a}$ \\
\hline & Salmonella typhimurium & 31,25 & $0,45^{\mathrm{a}}$ \\
\hline & Proteus mirabilis & $<3,9$ & $0,52^{a}$ \\
\hline \multirow{3}{*}{ Hongos } & Aspergillus brasiliensis & $<3,9$ & $0,25^{b}$ \\
\hline & Trichophytum rubrum & 125 & $2,1^{b}$ \\
\hline & $\begin{array}{l}\text { Trichophytum } \\
\text { mentagrophytes }\end{array}$ & 62,5 & $2,0^{b}$ \\
\hline Levaduras & Candida albicans & $<3,9$ & $0,41^{b}$ \\
\hline
\end{tabular}

${ }^{a}$ Gentamicina; ${ }^{b}$ Anfotericina B

encontrado en el aceite de P. odoratissimum, muestra una buena actividad frente a bacterias Gram negativas resistentes, como E. coli y P. aeruginosa. Lo anterior, es consistente con los resultados obtenidos en este estudio (Solórzano y Miranda, 2012). antimicrobiana. Sin embargo, los resultados de cada uno de los estudios mencionados, son obtenidos empleando diferentes métodos de evaluación y cepas microbianas, lo que dificulta la comparación de los mismos (Tavakoli et al, 2015). 
Los hallazgos de este estudio demuestran la fuerte actividad antimicrobiana del aceite esencial obtenido por hidrodestilación, a partir de las hojas y flores de $P$. odoratissimum, sobre todas las cepas microbianas ensayadas, actividad que se puede explicar por la presencia de compuestos como el geraniol, el citronelol, el linalol y el cariofileno, entre otros. Estos resultados sugieren que éste aceite esencial se puede considerar como una alternativa terapéutica para el tratamiento de enfermedades infecciosas asociadas a las cepas microbianas evaluadas. Es necesario realizar estudios de toxicidad que proporcionen información sobre la seguridad del aceite.

\section{AGRADECIMIENTOS}

Los autores expresan sus agradecimientos a la Fundación Universitaria Juan N. Corpas y a la Universidad Distrital Francisco José de Caldas.

\section{REFERENCIAS}

1. Adams, R. P. (2007). Identification of Essential Oil Components by Gas Chromatography / Mass Spectrometry. In Identification of Essential Oil Components by Gas Chromatography / Mass Spectrometry (4th Edition), Allured Publishing Corporation, Illinois, 401p.

2. Alviano DS, A. C. (2009). Plant extracts: Search for new alternatives to treat microbial diseases. Current Opinion in Biotechnology, 10, 106-121. http://doi.org/10.2174/138920109787 048607\#sthash.PuhINiAC.dpuf

3. Andrade, M. a., Cardoso, M. G., Batista, L. R., Freire, J. M., y Nelson, D. L. (2011). Antimicrobial activity and chemical composition of essential oil of Pelargonium odoratissimum. Brazilian Journal of Pharmacognosy, 21(1), 47-52. http:// doi.org/10.1590/S0102-695X2011005000009

4. Babushok, V., y Zenkevich, I. (2009). Retention Indices for most frequently reported essential oil compounds in GC. Chromatographia, 69, 257-269. http://doi:10.1063/1.3653552

5. Boukhatem, M. N., Kameli, A., y Saidi, F. (2013). Essential oil of Algerian rose-scented geranium (Pelargonium graveolens): Chemical composition and antimicrobial activity against food spoilage pathogens. Food Control, 34(1), 208-213. http://doi.org/10.1016/j. foodcont.2013.03.045

6. Bussmann, R. W., Malca, G., Glenn, a., Sharon, D., Chait, G., Díaz, D. y Benito, M. (2010). Minimum inhibitory concentrations of medicinal plants used in Northern Peru as antibacterial remedies. Journal of Ethnopharmacology, 132(1), 101-108. http://doi.org/10.1016/j. jep.2010.07.048 
7. Devi, K. P., Nisha, S. A., Sakthivel, R., \& Pandian, S. K. (2010). Eugenol (an essential oil of clove) acts as an antibacterial agent against Salmonella typhi by disrupting the cellular membrane. Journal of Ethnopharmacology, 130(1), 107-115. http://doi.org/10.1016/j. jep.2010.04.025

8. Goodner, K. L. (2008). Practical retention index models of OV-101, DB-1, DB-5, and DB-Wax for flavor and fragrance compounds. LWT Food Science and Technology, 41, 951-958. http://doi.org/10.1016/j.Iwt.2007.07.007

9. Langeveld, W. T., Veldhuizen, E. J. a, y Burt, S. (2014). Synergy between essential oil components and antibiotics: A review. Critical Reviews in Microbiology, 40(1), 76-94. http://doi. org/10.3109/1040841X.2013.763219

10. Lis-Balchin, M., Buchbauer, G., Ribisch, K., \& Wenger, M. T. (1998). Comparative antibacterial effects of novel Pelargonium essential oils and solvent extracts. Letters in Applied Microbiology, 27(3), 135-141. http:// DOI: 10.1046/j.1472-765X.1998.00414.x

11. Lorenzi, V., Muselli, A., Bernardini, A. F., Berti, L., Pagès, J. M., Amaral, L., y Bolla, J. M. (2009). Geraniol restores antibiotic activities against multidrug-resistant isolates from gramnegative species. Antimicrobial Agents and Chemotherapy, 53(5), 2209-2211. http://doi. org/10.1128/AAC.00919-08
12. Matusinsky, P., Zouhar, M., Pavela, R., y Novy, P. (2015). Antifungal effect of five essential oils against important pathogenic fungi of cereals. Industrial Crops and Products, 67, 208-215. http://doi.org/10.1016/j.indcrop.2015.01.022

13. Nurzyńska -Wierdak, R., Bogucka -Kocka, A., Kowalski, R, y Borowski, B. (2012). Changes in the chemical composition of the essential oil of sweet basil (Ocimum basilicum L.) depending on the plant growth stage. Chemija, 23(3), 216-222.

14. Radulovic, N. Blagojevic, P., y Palic, R. (2010). Comparative study of the leaf volatiles of $\mathrm{Ar}$ tostaphylos uva-ursi (L) Spreng and Vaccinium vitis-idaea L. (Ericaceae). Molecules, 15, 61686185. http:// doi:10.3390/molecules15096168

15. Romitelli, I. y Martins, M. (2013). Comparison of leaf morphology and anatomy among Malva sylvestris ("gerânio-aromático"), Pelargonium graveolens ("falsa-malva") and Pelargonium odoratissimum ("gerânio-de-cheiro"). Rev. Bras. PI. Med., Botucatu, 15(1), 91-97. http:// dx.doi.org/10.1590/S1516-05722013000100013

16. Solórzano-Santos, F., y Miranda-Novales, M. G. (2012). Essential oils from aromatic herbs as antimicrobial agents. Current Opinion in Biotechnology, 23(2), 136-141. http://doi. org/10.1016/j.copbio.2011.08.005

17. Tavakoli, H. R., Mashak, Z., Moradi, B., y Sodagari, H. R. (2015). Antimicrobial Activities of the Combined Use of Cuminum cyminum L. 
Essential Oil, Nisin and Storage Temperature Against Salmonella typhimurium and Staphylococcus aureus In Vitro. Jundishapur Journal of Microbiology, 8(4). http://doi.org/10.5812/ jjm.8(4)2015.24838

18. USP. (2014). USP Farmacopea de los Estados Unidos de America. (The United States Pharmacopeial Convention, Ed.) (37th ed.). Baltimore.

19. Vega, M., Verde, J., Oranday, A., Eufemia, M., Núñez, A., Rivera, A., y Benjamín, L. (2013). Actividad antibacteriana y citotóxica de Leucophyllum frutescens (Berl) I. M. Johnst del Norte de México contra from Northern Mexico against Staphylococcus aureus clinical isolates. Revista Mexicana de Ciencias Farmacéuticas. 44(2), 24-30.

20. WHO. 2014. Antimicrobial Resistance Global Report on Surveillance 2014. World Health Organization. (Francia): p. 231. http://reliefweb.int/sites/ reliefweb.int/files/resources/9789241564748_ eng.pdf, consulta Junio de 2015.

21. Williams, C., y Harborne, J. (2002). Phytochemistry of the genus Pelargonium. Geranium and Pelargonium, History of Nomenclature, Usage and Cultivation. En: Taylor \& Francis (Eds).p. 99-115.

22. Xianfei, X., Xiaoqiang, C., Shunying, Z., y Guolin, Z. (2007). Chemical composition and antimicrobial activity of essential oils of Chaenomeles speciosa from China. Food Chemistry, 100(4), 1312-1315. http://doi:10.1016/j. foodchem.2005.12.011
23. Zore, G. B., Thakre, A. D., Rathod, V., y Karuppayil, S. M. (2011). Evaluation of anti- Candida potential of geranium oil constituents against clinical isolates of Candida albicans differentially sensitive to fluconazole: Inhibition of growth, dimorphism and sensitization. Mycoses, 54(4), 99-109. htpp://doi: 10.1111/j.1439-0507.2009.01852.x 